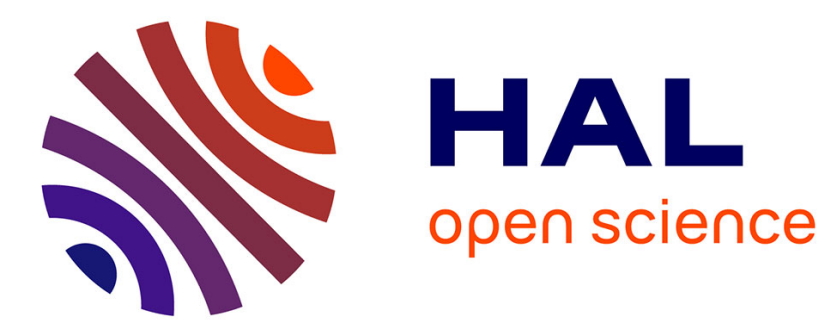

\title{
Class-jump phenomenon for physical symmetries in bi-dimensional space.
}

Nicolas Auffray, R. Bouchet, Y. Bréchet

\section{To cite this version:}

Nicolas Auffray, R. Bouchet, Y. Bréchet. Class-jump phenomenon for physical symmetries in bidimensional space.. Lecture Notes in Applied and Computational Mechanics, 46, Springer, pp.1-11, 2009, 10.1007/978-3-642-00911-2_1 . hal-00338839

\section{HAL Id: hal-00338839 \\ https://hal.science/hal-00338839}

Submitted on 14 Nov 2008

HAL is a multi-disciplinary open access archive for the deposit and dissemination of scientific research documents, whether they are published or not. The documents may come from teaching and research institutions in France or abroad, or from public or private research centers.
L'archive ouverte pluridisciplinaire HAL, est destinée au dépôt et à la diffusion de documents scientifiques de niveau recherche, publiés ou non, émanant des établissements d'enseignement et de recherche français ou étrangers, des laboratoires publics ou privés. 


\title{
Class-jump phenomenon for physical symmetries in bi-dimensional space.
}

\author{
N. Auffray ${ }^{1}, \mathrm{R}$. Bouchet ${ }^{1}$, and Y. Bréchet ${ }^{2}$ \\ 1 ONERA/DMSM, 29 Avenue de la Division Leclerc, F-92322, Châtillon Cedex, France \\ nicolas .auffray@onera.fr \\ 2 LTPCM, BP 75, Domaine Universitaire de Grenoble, F-38402, Saint Martin d'Hères Cedex, France
}

In condensed matter physics tensors are used to model physical properties of materials. Some well known examples are elasticity which is modeled by a $4 t h$-order tensor or piezoelectricity by a $3 \mathrm{rd}-$ order tensor. Some tensors of higher order might occur in theory of generalized continuum, for example in Mindlin's strain-gradient elasticity [2] in which the physical state of the material is described in each point using three tensors from $4 t h$-order to $6 t h$-order one. Damage could also be modeled by an $8 t h$-order tensor. When ones studying such complex models the first step is to focus attention in its bi-dimensional version. Taking a complex problem at a lower level help to better understand the physics of the behavior. But we have to wonder in which measure the phenomenon we study is independent of the dimension of the space we are working in. In this paper we will focus our attention on the issue of symmetry classes of linear constitutive behavior, and show that in a two dimension anomalies could occur.

\section{Physical and material symmetries}

In the following $\mathcal{E}^{d}$ will be the $d$-dimensional euclidean space, $\mathcal{E}^{d}$ will represent the space we are working in. We will consider the cases $d=2$ and $d=3$.

Let $\mathrm{G}$ be a group of operations, a material $\mathcal{M}$ is said to be $\mathrm{G}$-invariant if the action of the elements of $\mathrm{G}$ transform $\mathcal{M}$ into itself. We shall note $\mathrm{G}_{\mathcal{M}}$ this set of operation, namely the material symmetry group, and defined

$$
\mathrm{G}_{\mathcal{M}}=\{\mathrm{Q} \in \mathrm{O}(d), \quad \mathrm{Q} \star \mathcal{M}=\mathcal{M}\}
$$

where $\star$ represents the action of $\mathrm{Q}$ upon $\mathcal{M}$ and $\mathrm{O}(d)$ is the orthogonal group in $d$ dimension.

Consider now a physical property $\mathcal{P}$ defined on a material. The physical group of symmetry is defined as the set of operations that leaves the behavior invariant. We will note $G_{\mathcal{P}}$ this group defined as

$$
\mathrm{G}_{\mathcal{P}}=\{\mathrm{Q} \in \mathrm{O}(d), \quad \mathrm{Q} \star \mathcal{P}=\mathcal{P}\}
$$

We will suppose now that the physical property could be described by an $n$-order tensor, we will note $\mathrm{T}^{(n)}$ this tensor and $\mathbb{T}^{(n)}$ the related vector space. The action of $\mathrm{O}(d)$ on $\mathrm{T}^{(n)}$ is defined by the mean of the Rayleigh product: 


$$
\star: \mathrm{O}(d) \times \mathbb{T}^{(n)} \rightarrow \mathbb{T}^{(n)}:\left(\mathrm{Q}, \mathrm{T}^{(n)}\right) \mapsto \mathrm{Q} \star \mathrm{T}^{(n)}:=Q_{i_{1} j_{1}} \ldots Q_{i_{n} j_{n}} T_{j_{1} \ldots j_{n}}^{(n)}
$$

So the group of symmetry of a tensor $\mathrm{T}^{(n)}$ is the set of elements in $\mathrm{O}(d)$ :

$$
\mathrm{G}_{T^{(n)}}=\left\{\mathrm{Q} \in \mathrm{O}(d) \mid Q_{i_{1} j_{1}} \ldots Q_{i_{n} j_{n}} T_{j_{1} \ldots j_{n}}^{(n)}=T_{i_{1} \ldots i_{n}}^{(n)}\right\}
$$

According to the different symmetry properties of its elements the space $\mathbb{T}^{(n)}$ is divided in equivalence classes [3].

The material symmetry group and the physical one are related by the mean of Neumann's principle [4]. In this way we get the inclusion:

$$
\mathrm{G}_{\mathcal{M}} \subseteq \mathrm{G}_{\mathcal{P}}
$$

This relation means that every operation that leaves the material invariant let the physical properties invariant. Nevertheless, as shown for tensorial properties by Hermann's theorems [6], physical properties could be more symmetrical than the material.

In $\mathcal{E}^{2}$ plane and spatial material symmetry group coincide meanwhile there are distinct in $\mathcal{E}^{3}$. In case of plane invariance $\mathrm{G}_{\mathcal{M}}$ should be conjugate to a subgroup of $\mathrm{O}(2)$ [4]. The collection of those subgroups is [3]

$$
\Sigma:=\left\{I, Z_{p}, D_{p}, \mathrm{SO}(2), \mathrm{O}(2)\right\}
$$

Where $I$ is the identity group, $Z_{p}$ is the cyclic group of order $p$, it is the group of rotation of a chiral figure that possesses an $p$-fold axe of invariance. $D_{p}$ is the dihedral group of order $2 p$, it is the group of operations that leave a regular $p$-gone invariant. $D_{p}$ contains $Z_{p}$ and mirror symmetry. $\mathrm{SO}(2)$ is the continuous group of rotations and $\mathrm{O}(2)$ is the continuous group of orthogonal transformations in two dimension. In the sequel we will often refer to rotational invariance, according to formers definitions it means the collection of SO2-subgroups.

To study the symmetry classes of tensors a decomposition in elementary elements is needed. Such a decomposition is referred in literature as the harmonic decomposition $[3,1]$ or the irreducible decomposition $[8,7]$.

\section{Harmonic Decomposition}

\subsection{Generality}

We call harmonic decomposition the orthogonal irreducible decomposition of a tensor. In $\mathcal{E}^{d}$ this decomposition is $\mathrm{O}(d)$-invariant. This decomposition is well known in group representation theory and allow us to decompose a tensor of any finite order as a sum of irreducible ones $[7,8]$. And so

$$
\mathrm{T}^{(n)}=\sum_{k, \tau} \mathrm{D}(n)^{k, \tau}
$$

Tensors $\mathrm{D}(n)^{k, \tau}$ are components of the irreducible decomposition, $k$ denote the order of the harmonic tensor embedded in $\mathrm{D}(n)$ and $\tau$ separate terms of same order. This decomposition establish an isomorphism between $\mathbb{T}^{(n)}$ and a direct sum of harmonic tensor vector spaces $\mathbb{H}^{k}[3]$. We shall note 


$$
\mathbb{T}^{(n)}=\bigoplus_{k, \tau} \mathbb{H}^{k, \tau}
$$

but as explained in [1] this decomposition is not unique. But the following decomposition, which groups terms of same order, is unique and is called the $\mathrm{O}(d)$-isotypic decomposition.

$$
\mathbb{T}^{(n)}=\bigoplus_{k=0}^{n} \alpha_{k} \mathbb{H}^{k}
$$

where $\alpha_{k}$ is the multiplicity of $\mathbb{H}^{k}$ in the decomposition. Harmonic tensors are totally symmetric and traceless, and the dimension of the associated vector spaces are:

$$
\operatorname{dim} \mathbb{H}^{k}=\left\{\begin{array}{l}
2 k+1, \quad \forall k \geq 0 \text { in } 3 D \\
2, \quad \forall k>0, \text { and } 1 \text { if } k=0 \text { in } 2 D
\end{array}\right.
$$

For the sake of simplicity when there is no risk of misunderstanding we will note $K^{\alpha_{k}}$ spaces $\alpha_{k} \mathbb{H}^{k}$. That means the order $K$ of the subspace along with its multiplicity $\alpha_{k}$. Moreover, as we are dealing with $2 D$ and $3 D$ tensor spaces, we will distinguish in the notation the two kind of vector space with an $*$ exponent for the bi-dimensional one. We shall note, for example, the bi-dimensional harmonic decomposition of $\mathbb{T}^{*(n)}$

$$
\mathbb{T}^{*(n)}=\bigoplus_{k=0}^{n} \alpha_{k}^{*} \mathbb{H}^{* k}
$$

The families $\left\{\alpha_{k}\right\}$ and $\left\{\alpha_{k}^{*}\right\}$ are function of the order of the tensor space and of its index symmetries. There exist several methods to compute those different families, more details can be found in $[8,7,5]$. A general result on the structure of $2 D$ and $3 D$ harmonic decomposition can be find in [7]. For a generic tensor, a tensor space with no index symmetry, the two following theorems hold true:

Theorem 1. Let $\mathbb{T}^{*(n)}$ be a $2 D$ generic $n$-order tensor vector space. If $n=2 q$ its harmonic decomposition only contains subspaces of even order, and if $n=2 q+1$ its harmonic decomposition only contains subspaces of odd order.

Theorem 2. Let $\mathbb{T}^{(n)}$ be a $3 D$ generic n-order tensor. Its harmonic decomposition contains subspaces of $k$-order for any $k$ with $0 \leq k \leq n$

Taking into account index symmetries can make odd or even components to vanish in the $3 D$ cases, for example a full symmetrical tensor of even order would just contain harmonic tensor of even order and reciprocally for full symmetrical odd tensor [8].

A classical example is the $3 D$ space of elasticity tensors [3] ; this space is isomorphic to $0^{2} \oplus 2^{2} \oplus 4$ [3] which is a 21 dimensional vector space. In $2 D$ the same space will be isomorphic to $0^{* 2} \oplus 2^{*} \oplus 4^{*}$ [7]. This space is a 6 dimensional vector space. The information provided by those two decompositions does not allow one to make a direct comparison of theirs structures. In order to do that we should express the $\mathrm{O}(2)$-isotypic decomposition of $\mathbb{T}^{(n)}$.

We will now focus on the link between $3 D$ harmonic decomposition and $2 D$ one. Our aim is, for rotational action, to establish an isomorphism between $3 D$ vector spaces and a direct sum of $2 D$ harmonic tensor vector spaces. 


\subsection{Cartan decomposition}

As shown and demonstrated in [1] under the action of $\mathrm{O}(2)$ the space $\mathbb{H}^{k}$ admits the following decomposition:

$$
\mathbb{H}^{k}=\bigoplus_{j=0}^{k} \mathbb{K}_{j}^{k}
$$

where

$$
\operatorname{dim} \mathbb{K}_{j}^{k}=\left\{\begin{array}{l}
1 \text { if } j=0 \\
2 \text { if } j \neq 0
\end{array}\right.
$$

This decomposition is referred as the Cartan's decomposition of harmonic tensor space. The relation (12) implies a decomposition that contains subspaces for each $j$ between 0 and $k$.

For $(x, y, z) \in \mathbb{R}^{3}$ let $w=x+i y$ be the plane complex vector with $i=\sqrt{-1}$. As explained in [1] the space $\mathbb{K}_{j}^{k}$ is spanned by

$$
k_{l}^{j}=\left\{k_{1}^{j}, \quad k_{2}^{j}\right\}=\left\{z^{k-j} \Re\left(w^{j}\right), z^{k-j} \Im\left(w^{j}\right)\right\}
$$

where $\Re, \Im$ are functions returning the real and imaginary part of a complex number. In the same time the space $\mathbb{H}^{* j}$ is spanned [7] by

$$
h_{l}^{j}=\left\{h_{1}^{j}, \quad h_{2}^{j}\right\}=\left\{\Re\left(w^{j}\right), \Im\left(w^{j}\right)\right\}
$$

So if $z \neq 0$ for each space $\mathbb{K}_{j}^{k}$ there exists a bijective function $\phi_{j}^{k}$ that turns $\mathbb{K}_{j}^{k}$ basis vectors into $\mathbb{H}^{* j}$ ones. $\phi_{j}^{k}$ is defined as

$$
\phi_{j}^{k}\left(k_{l}^{j}\right)=\frac{k_{l}^{j}}{z^{k-j}}=h_{l}^{j}
$$

So by the family $\left\{\phi_{j}^{k}\right\}_{0 \leq j \leq k}$ the space $\mathbb{H}^{k}$ is isomorphic to the following space:

$$
\mathbb{H}^{k}=\bigoplus_{j=0}^{k} \mathbb{H}^{* j}
$$

So we may rewrite the relation (9)

$$
\mathbb{T}^{(n)}=\bigoplus_{k=0}^{n} \alpha_{k}\left(\bigoplus_{j=0}^{k} \mathbb{H}^{* j}\right)=\bigoplus_{k=0}^{n} \sum_{j=k}^{n} \alpha_{j} \mathbb{H}^{* k}=\bigoplus_{k=0}^{n} \beta_{k} \mathbb{H}^{* k}
$$

This decomposition is the $\mathrm{O}(2)$-isotypic decomposition of a three dimensional tensor. We obtain the following lemma for the planar decomposition of an $3 D$ operator:

Lemma 1. Let $\mathbb{T}^{(n)}$ be a $3 D$ n-order tensor vector space with any kind of indicial symmetry. Its planar harmonic decomposition contains subspaces of $k$-order for any $k$ within $0 \leq k \leq n$

Proof. The planar decomposition is written

$$
\mathbb{T}^{(n)}=\bigoplus_{k=0}^{n} \beta_{k} \mathbb{H}^{* k}
$$


So to demonstrate this lemma we shall prove the following property

$$
\forall k \in[0, n], \beta_{k}>0
$$

The proof is straight forward. Let's consider the harmonic decomposition of a tensor space $\mathbb{T}^{(n)}$. Regardless of the index symmetry of that space we got a term of order $n$ in this decomposition. By property of harmonic decomposition the multiplicity of that harmonic space must be of 1 . By the mean of Cartan's decomposition this space will be decomposed into $n+1$ Cartan's subspaces. We got

$$
\forall k \in[0, n], \beta_{k}=\sum_{i=k}^{n} \alpha_{i}=1+\sum_{i=k}^{n-1} \alpha_{i}
$$

and $\alpha_{i} \in \mathbb{N}$ we can conclude that

$$
\forall k \in[0, n], \beta_{k} \geq 1
$$

which concludes the proof.

So theorems 1 and lemma 1 allow us to formulate the following theorem:

Theorem 3. Let $\mathbb{T}^{(n)}$ be the any nth-order tensor vector space. In $\mathcal{E}^{3}$ its planar harmonic spectrum will be full meanwhile in $\mathcal{E}^{2}$ it would be sparse according to the parity of $n$.

If we come back to the example of elasticity, we remind that, in $3 D$, its vector space is isomorphic to $0^{2} \oplus 2^{2} \oplus 4$. According to the formula given in (18) its planar decomposition would be $0^{* 5} \oplus 1^{* 3} \oplus 2^{* 3} \oplus 3^{*} \oplus 4^{*}$. This decomposition is the one to compare with the bi-dimensional harmonic decomposition which is $0^{* 2} \oplus 2^{*} \oplus 4^{*}$.

This difference in the composition of the planar spectrum will led to different systems of symmetry in $2 D$ and $3 D$ physical spaces. Let's show that with the study of the rotational-invariance systems of symmetry.

\section{Rotational invariance}

Let $\mathrm{G}_{\mathrm{T}^{n}}$ be the group of operations that leaves $\mathrm{T}^{n}$ unchanged, i.e.:

$$
\mathrm{Q} \in \mathrm{G}_{\mathrm{T}^{n}} \Rightarrow \mathrm{Q} \star \mathrm{T}^{n}=\mathrm{T}^{n}
$$

The space $\mathbb{T}^{n}$ is isomorphic with $\bigoplus_{k=0}^{n} \beta_{k} \mathbb{H}^{* k}$. So any $\forall \mathrm{T}^{n} \in \mathbb{T}^{n}$ is defined by a family of tensors $\left\{\mathrm{H}^{* k, \tau}\right\}$. The order of this family is obviously $m=\sum_{k=0}^{n} \beta_{k}$. As this decomposition is $\mathrm{SO}(2)$ invariant the invariance condition on $\mathrm{T}^{n}$ could be expressed as $m$ conditions on the elements of the planar decomposition. Those $m$ conditions are of $n+1$ different types according to the order of the bi-dimensional harmonic tensor, that means

$$
\mathrm{Q} \star_{k} \mathrm{H}^{* k}=\mathrm{H}^{* k}
$$

where $\star_{k}$ is the action of $\mathrm{SO}(2)$ on $\mathbb{H}^{* k}$, this action shall easily be expressed in the sequel.

Let $\mathrm{H}^{* k}=\left(s_{k}, t_{k}\right)$ be a vector of a space $\mathbb{H}^{* k}$. Consider a plane rotation $\mathrm{Q}_{\text {rot }} \in \mathrm{SO}(2)$, for example take the generator of $Z_{p}$, we got 


$$
\mathrm{Q}_{\text {rot }}=\left(\begin{array}{cc}
\cos \frac{2 \pi}{p} & -\sin \frac{2 \pi}{p} \\
\sin \frac{2 \pi}{p} & \cos \frac{2 \pi}{p}
\end{array}\right)
$$

As shown in [1] $\mathrm{Q}_{\text {rot }}$ act on $\mathbb{H}^{* k}$ as a generator of $Z_{\frac{p}{k}}$, the order of the rotation $p$ is divided by the indice of Cartan's subspace:

$$
\mathrm{Q}_{\text {rot }} \star \mathrm{H}^{* k}=\left(\begin{array}{cc}
\cos \frac{2 k \pi}{p} & -\sin \frac{2 k \pi}{p} \\
\sin \frac{2 k \pi}{p} & \cos \frac{2 k \pi}{p}
\end{array}\right)\left(\begin{array}{l}
s_{k} \\
t_{k}
\end{array}\right)
$$

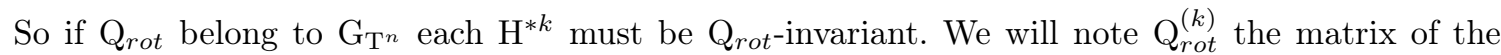
action of $\mathrm{Q}_{\text {rot }}$ on $\mathrm{H}^{* k}$, the invariance condition of $\mathrm{H}^{* k}$ is simply the solution of $\left(\mathrm{Q}_{\text {rot }}^{(k)}-\mathrm{Id}\right) \mathrm{H}^{* k}=0$. In other word we have to study $\operatorname{ker}\left(\mathrm{Q}_{r o t}^{(k)}-\mathrm{Id}\right)$. A direct calculation shows that the condition of invariance of $\mathrm{H}^{* k}$ under the $Z_{p}$-action is:

$$
k=t p, \quad t \in \mathbb{N}
$$

That mean that if $k \neq t p$ then $\mathrm{H}^{* k}$ equal 0 . So the planar decomposition of a $Z_{p}$-invariant space $\mathbb{T}^{(n)}, Z_{p} \mathbb{T}^{n}$, will be:

$$
Z_{p} \mathbb{T}^{n}=\bigoplus_{0 \leq m \leq\left\lfloor\frac{n}{p}\right\rfloor} \beta_{m p} \mathbb{H}^{* m p}
$$

where $\lfloor$.$\rfloor is the floor function. It is clear that in the case of p>n$ the decomposition (28) will reduced to

$$
Z_{p} \mathbb{T}^{n}=\beta_{0} \mathbb{H}^{* 0}
$$

wich only contains the hemitropic components. The relation (28) allow us to compare consequences of material invariance on operator in different cases.

For any three dimensional tensor we got as a consequence of the theorem 1 the following theorem

Theorem 4. Let's consider the vector space $\mathbb{T}^{n}$ in $3 D$. Non-equivalent rotational material invariance of order lower than or equal to $n$ lead to distinct anisotropic classes for the tensorial properties.

Proof. As we are dealing with a tensorial vector space in $3 D$ we know that its planar decomposition is complete. For any $p$ and $q$ greater than $n$ we got $Z_{p} \mathbb{T}^{n}={ }^{Z_{q}} \mathbb{T}^{n}=\beta_{0} \mathbb{H}^{* 0}$ and so we conclude that this condition lead the same anisotropic class: transverse hemitropy ${ }^{3}$. This property is related to Hermann's theorems [5]. Now suppose that $p$ and $q$ are both less than or equal to $n$. Let's search $p, q \in \mathbb{N}^{* 2}$ that verify ${ }_{p} \mathbb{T}^{n}={ }^{Z_{q}} \mathbb{T}^{n}$ for a given vector space $\mathbb{T}^{n}$.

$$
\bigoplus_{0 \leq m \leq\left\lfloor\frac{n}{p}\right\rfloor} \beta_{m p} \mathbb{H}^{* m p}=\bigoplus_{0 \leq m^{\prime} \leq\left\lfloor\frac{n}{q}\right\rfloor} \beta_{m^{\prime} q} \mathbb{H}^{* m^{\prime} q}
$$

this relation implies that the following indices equality hold: $m p=m^{\prime} q,\left\{m, m^{\prime}, p, q\right\} \in \mathbb{N}^{4}$.

$$
\forall m, \exists m^{\prime}, m^{\prime}=m \frac{p}{q}
$$

\footnotetext{
${ }^{3}$ Mirror invariance should combine with the rotational one to obtain the transverse isotropy class.
} 
and so $\frac{p}{q}=k, k \in \mathbb{N}^{*}$. Reciprocally

$$
\forall m^{\prime}, \exists m, m=m^{\prime} \frac{q}{p}
$$

and so $\frac{q}{p}=k^{\prime}=\frac{1}{k}, k^{\prime} \in \mathbb{N}^{*}$, which implies that $k$ must be 1 . The only solution is $p=q$, so reciprocally $p \neq q$ implies $Z_{p} \mathbb{T}^{n} \neq Z^{Z_{q}} \mathbb{T}^{n}$.

As an example of what this property implies let's consider a 6 th-order tensor A. For a physical illustration this tensor could be the second order elasticity that appears in theory of strain-gradient elasticity [2]. By the mean of Hermann's theorems [6] we know that for any material invariance of order greater that 6 our tensor will be transverse hemitropic. By the mean of the theorem 4 we know that any other rotational invariance lead to distinct classes of symmetry. And so, if we just consider the subgroup of $\mathrm{SO}(2)$, we got 7 different rotational invariant type of tensors: $\left\{I, Z_{2}, Z_{3}, Z_{4}, Z_{5}, Z_{6}, \mathrm{SO}(2)\right\}$. Let's consider now the same problem in a two dimensional physical space.

\section{Bi-dimensional physical space}

To study the plane invariance in $3 D$ we completed the use of the harmonic decomposition by the Cartan's one. In $2 D$ the problem is simpler and we don't have to decompose the vector space $\mathbb{T}^{*(n)}$ any further. The action on $\mathrm{Q}_{\text {rot }}$ on the $2 D$ harmonic decomposition is the same as the one we introduce for the Cartan decomposition in the $3 D$ case and all the things we said about the condition of invariance remain the same. The only difference arises from the theorem 1: in $2 D$ the harmonic decomposition of an even-order tensor just contain even-order and reciprocally. This property will generate a phenomenon, we call "Class-Jump", that makes some physical properties more symmetrical that they should.

\subsection{Even order tensor}

Consider first the case of the vector space $\mathbb{T}^{*(2 q)}$ in $2 D$, according to theorem 1 its $\mathrm{O}(2)$-isotypic decomposition would be written:

$$
\mathbb{T}^{*(2 q)}=\bigoplus_{k=0}^{q} \alpha_{2 k}^{*} \mathbb{H}^{* 2 k}
$$

Consider the sub-set of $Z_{p}$-invariant tensors, for those tensor the non-vanishing coefficients would belong to subspace of order $2 k$ verifying the relation:

$$
2 k=t p, \quad t \in \mathbb{N}
$$

We now have to consider two cases: $p=2 q^{\prime}$ and $p=2 q^{\prime}+1$. Suppose first $p=2 q^{\prime}$, we obtain the classical condition $k=t q^{\prime}$. But if we consider the case $p=2 q^{\prime}+1$ we obtain:

$$
2 k=t\left(2 q^{\prime}+1\right), \quad t \in \mathbb{N}
$$

The former relation make sense only for $t=2 t^{\prime}$, and so we obtain 


$$
2 k=t^{\prime}\left(2\left(2 q^{\prime}+1\right)\right), \quad t^{\prime} \in \mathbb{N}
$$

Which is the same restriction as the one imposed by an $Z_{\left(2\left(2 q^{\prime}+1\right)\right)^{-}}$material invariance. A $Z_{\left(2 q^{\prime}+1\right)^{-}}$ material invariance will impose the same restriction on the tensors as a $Z_{\left(2\left(2 q^{\prime}+1\right)\right)^{-}}$invariance. This demonstrate the following theorem:

Theorem 5. In a 2D, an even order tensor could not see an odd-order material invariance, the invariance seen would be twice the order of the former.

Now consider the case of odd-order tensors.

\subsection{Odd order tensor}

According to theorem 1, the $\mathrm{O}(2)$-isotypic decomposition of the vector space $\mathbb{T}^{*(2 q+1)}$ would be written:

$$
\mathbb{T}^{*(2 q+1)}=\bigoplus_{k=0}^{q} \alpha_{2 k+1}^{*} \mathbb{H}^{* 2 k+1}
$$

Consider the sub-set of $Z_{p}$-invariant tensors, for those tensor the non-vanishing coefficients would belong to subspace of order $2 k+1$ verifying the relation:

$$
(2 k+1)=t p, \quad t \in \mathbb{N}
$$

We consider first the case $p=2 q^{\prime}$, we have

$$
(2 k+1)=2 t q^{\prime}, \quad t \in \mathbb{N}
$$

As $t \in \mathbb{N}$ the former relation could never be satisfied. And so we demonstrate the following theorem

Theorem 6. In a $2 D$ an odd tensor could not see an even-order material invariance, the tensor would vanish for that kind of material invariance.

Consider now $p=2 q^{\prime}+1$ we got the relation

$$
(2 k+1)=t\left(2 q^{\prime}+1\right), \quad t \in \mathbb{N}
$$

If we suppose $t$ to be even we will find the relation (39), and so we conclude that there is no solution for $t=2 t^{\prime}$. Suppose now $t=2 t^{\prime}+1$, we obtain:

$$
(2 k+1)=(2 t+1)\left(2 q^{\prime}+1\right)
$$

and so we obtain a relation of the form $k^{\prime}=t^{\prime} r^{\prime}$ for $\left\{k^{\prime}, t^{\prime}, r^{\prime}\right\} \in(2 \mathbb{N}+1)$. In this case we don't have any specific behavior for the symmetry classes. Distinct material invariances will lead to distinct tensorial classes. 


\subsection{Synthesis}

We can sum up all the results we obtain for rotational invariance in two dimensional space in the following array:

\begin{tabular}{|c||c|c|}
\hline & $\mathbb{T}^{*(2 k)}$ & $\mathbb{T}^{*(2 k+1)}$ \\
\hline \hline$Z_{2 p}$ & $t \in \mathbb{N}$ & $t=\emptyset$ \\
\hline$Z_{2 p+1}$ & $t \in(2 \mathbb{N}) \Rightarrow$ jump to $Z_{2(2 p+1)}$ & $t \in(2 \mathbb{N}+1)$ \\
\hline
\end{tabular}

The point to stress are the class jump phenomenon for an odd material invariance in the case of a even tensor, and the vanishing effect for the opposite case. A demonstrated by theorem 4, none of these effect could be seen in three-dimensional space.

Let's go back to the example of a 6 th-order tensor. We show in $\S .3$ that in three dimensional space we got 7 different types of rotational invariance. By the mean of theorem 5 we claim that such a tensor in a two dimensional space could only belong to 4 different systems ${ }^{4}:\left\{Z_{2}, Z_{4}, Z_{6}, \mathrm{SO}(2)\right\}$.

\section{Conclusion}

We show in that paper that the symmetry class of any tensorial property depends on the space dimension. In $2 D$ some jump of symmetry could occur making physical properties more symmetrical than the material they are defined on. This point is related to the reciprocity of Hermann's theorems [6]. Those theorems state that if the order of the rotational invariance exceed the number of indexes of a tensor then this tensor is, a least, transverse hemitropic ( $\mathrm{SO}(2)$-invariant). For the $3 D$ case theorems 1 and 4 show that the reciprocity is true. But as corollary of theorem 5 the reciprocity fail for a bi-dimensional space. If we consider a $6 t h$-order tensor then a $Z_{5}$-material invariance will be seen as a $Z_{10}$-one by the tensor and so we can conclude that $Z_{5}$-material invariance imply a 6 th-order tensor to be at least hemitropic. And so a continuous class of symmetry could appear for an order of rotation lower than the number of index of our tensor.

\section{References}

1. Golubitsky M, Stewart I, Schaeffer D (1989) Singularities and Groups in Bifurcation Theory. Volume II. Springer-Verlag

2. Mindlin R, Eshel N (1968) Int. J. Solids Struct 4:109-124

3. Forte S, Vianello M (1996) J. Elastic. 43:81-108

4. Zheng Q, Boehler J (1994) Acta Mecha. 102:73-89

5. Auffray N (2008) CR. Meca. 336:458-463

6. Auffray N (2008) CR. Meca. 336:370-375

7. Zou W, Zheng Q, Du D, Rychlewski J (2001) Math. Mech. Solids 6:249-267

8. Jerphagnon J, Chemla D, Bonneville R (1978) Adv. Physics. 27:609-650

\footnotetext{
${ }^{4}$ It should be note that in $2 D$ a physical property defined on a material with no symmetry is $Z_{2}$-invariant.
} 